\title{
Spin Kostka polynomials
}

\author{
Jinkui Wan · Weiqiang Wang
}

Received: 23 February 2011 / Accepted: 13 March 2012 / Published online: 24 March 2012

(C) Springer Science+Business Media, LLC 2012

\begin{abstract}
We introduce a spin analogue of Kostka polynomials and show that these polynomials enjoy favorable properties parallel to the Kostka polynomials. Further connections of spin Kostka polynomials with representation theory are established.
\end{abstract}

Keywords Kostka polynomials - Symmetric groups · Schur $Q$-functions · Hall-Littlewood functions $\cdot q$-weight multiplicity $\cdot$ Hecke-Clifford algebra

\section{Introduction}

\section{1}

The Kostka numbers and Kostka(-Foulkes) polynomials are ubiquitous in algebraic combinatorics, geometry, and representation theory. A most interesting property of Kostka polynomials is that they have non-negative integer coefficients (due to Lascoux and Schützenberger [11]), and this has been derived by Garsia and Procesi [5] from Springer theory of Weyl group representations [18]. Kostka polynomials also coincide with Lusztig's $q$-weight multiplicity in finite-dimensional irreducible representations of the general linear Lie algebra [9, 12]. R. Brylinski [1] introduced a Brylinski-Kostant filtration on weight spaces of finite-dimensional irreducible representations and proved that Lusztig's $q$-weight multiplicities (and hence Kostka polynomials) are precisely the polynomials associated to such a filtration. For more on

\footnotetext{
J. Wan $(\bowtie)$

Department of Mathematics, Beijing Institute of Technology, Beijing, 100081, PR China e-mail:wjk302@gmail.com
}

W. Wang

Department of Mathematics, University of Virginia, Charlottesville, VA 22904, USA

e-mail:ww9c@virginia.edu 
Kostka polynomials, we refer to Macdonald [13] or the survey paper of Désarménien, Leclerc and Thibon [3].

The classical theory of representations and characters of symmetric groups admits a remarkable spin generalization due to Schur [15]. Many important constructions for symmetric groups and symmetric functions admit highly nontrivial spin counterparts, including Schur $Q$-functions and shifted tableaux (cf. e.g. [13]) and RobinsonShensted-Knuth correspondence (see Sagan [14]).

The goal of this paper is to add several items to the list of spin counterparts of classical theory. We introduce a notion of spin Kostka polynomials, and establish their main properties including the integrality and positivity as well as representation theoretic interpretations. We also introduce a notion of spin Hall-Littlewood polynomials. Our constructions afford natural $q, t$-generalizations in connection with Macdonald polynomials. The definitions made in this paper look very classical, and we are led to them from representation theoretic considerations. Once things are set up right, the proofs of the main results, which are based on the classical deep work on Kostka polynomials, are remarkably easy.

There has been a very interesting work of Tudose and Zabrocki [20] who defined a version of spin Kostka polynomials and spin Hall-Littlewood polynomials (in different terminology), adapting the vertex operator technique developed by Jing [7] and others (cf. Shimozono and Zabrocki [17]). Their definitions do not coincide with ours as shown by examples, and the precise connection between the two (if it exists) remains unclear. The $Q$-Kostka polynomials of Tudose and Zabrocki conjecturally admit integrality and positivity, but their approach does not seem to easily exhibit the connections to representation theory or afford $q, t$-generalization as developed in this paper. Throughout the paper we work with the complex field $\mathbb{C}$ as the ground field.

Denote by $\mathcal{P}$ the set of partitions and by $\mathcal{P}_{n}$ the set of partitions of $n$. Denote by $\mathcal{S P}$ the set of strict partitions and by $\mathcal{S} \mathcal{P}_{n}$ the set of strict partitions of $n$. Let $\Lambda$ denote the ring of symmetric functions in $x=\left(x_{1}, x_{2}, \ldots\right)$, and let $\Gamma$ be the subring of $\Lambda$ with a $\mathbb{Z}$-basis given by the Schur $Q$-functions $Q_{\xi}(x)$ indexed by $\xi \in \mathcal{S P}$, cf. [13].

As an element in the ring $\Lambda$, the Schur $Q$-functions $Q_{\xi}(x)$ can be expressed as a linear combination in the basis of the Hall-Littlewood functions $P_{\mu}(x ; t)$ and we define the spin Kostka polynomial $K_{\xi \mu}^{-}(t)$, for $\xi \in \mathcal{S P}$ and $\mu \in \mathcal{P}$, to be the corresponding coefficient; see (2.7). Recall that the entries $K_{\lambda \mu}(t), \lambda, \mu \in \mathcal{P}$ of the transition matrix from the Schur basis $\left\{s_{\lambda}\right\}$ to the Hall-Littlewood basis $\left\{P_{\mu}(x ; t)\right\}$ for $\mathbb{Z}[t] \otimes_{\mathbb{Z}} \Lambda$ are the Kostka polynomials.

Our first result concerns some remarkable properties satisfied by the spin Kostka polynomials (compare with Theorem 2.1, where some well-known properties of the usual Kostka polynomials are listed). For a partition $\lambda \in \mathcal{P}$ with length $\ell(\lambda)$, we set

$$
n(\lambda)=\sum_{i \geq 1}(i-1) \lambda_{i}, \quad \delta(\lambda)= \begin{cases}0, & \text { if } \ell(\lambda) \text { is even } \\ 1, & \text { if } \ell(\lambda) \text { is odd }\end{cases}
$$


For $\xi \in \mathcal{S P}$, we denote by $\xi^{*}$ the shifted diagram of $\xi$, by $c_{i j}$ the content, by $h_{i j}^{*}$ the shifted hook length of the cell $(i, j) \in \xi^{*}$. Also let $K_{\xi \mu}^{-}$be the number of marked shifted tableaux of shape $\xi$ and weight $\mu$; see Sect. 2.2 for precise definitions.

Theorem A The spin Kostka polynomials $K_{\xi \mu}^{-}(t)$ for $\xi \in \mathcal{S P}_{n}, \mu \in \mathcal{P}_{n}$ have the following properties:

(1) $K_{\xi \mu}^{-}(t)=0$ unless $\xi \geq \mu ; K_{\xi \xi}^{-}(t)=2^{\ell(\xi)}$.

(2) The degree of the polynomial $K_{\xi \mu}^{-}(t)$ is $n(\mu)-n(\xi)$.

(3) $2^{-\ell(\xi)} K_{\xi \mu}^{-}(t)$ is a polynomial with non-negative integer coefficients.

(4) $K_{\xi \mu}^{-}(1)=K_{\xi \mu}^{-} ; K_{\xi \mu}^{-}(-1)=2^{\ell(\xi)} \delta_{\xi \mu}$.

(5) $K_{(n) \mu}^{-}(t)=t^{n(\mu)} \prod_{i=1}^{\ell(\mu)}\left(1+t^{1-i}\right)$.

(6) $K_{\xi\left(1^{n}\right)}^{-}(t)=\frac{t^{n(\xi)}(1-t)\left(1-t^{2}\right) \cdots\left(1-t^{n}\right) \prod_{(i, j) \xi \xi^{*}}\left(1+t^{c_{i j}}\right)}{\prod_{(i, j) \in \xi^{*}}\left(1-t^{h_{i j}^{*}}\right)}$.

It is known (cf. Kleshchev [10]) that the spin representation theory of the symmetric group is equivalent to its counterpart for Hecke-Clifford algebra $\mathcal{H}_{n}:=\mathcal{C}_{n} \rtimes \mathbb{C} S_{n}$, and the irreducible $\mathcal{H}_{n}$-(super)modules $D^{\xi}$ are parameterized by strict partitions $\xi \in$ $\delta \mathcal{P}_{n}$. The Hecke-Clifford algebra $\mathcal{H}_{n}$ as well as its modules in this paper admit a $\mathbb{Z}_{2}$-graded (i.e, super) structure even though we will avoid using the terminology of supermodules.

For a partition $\mu \in \mathcal{P}_{n}$, let $\mathcal{B}_{\mu}$ be the variety of flags preserved by a nilpotent matrix of Jordan block form of shape $\mu$, which is a closed subvariety of the flag variety $\mathcal{B}$ of $G L_{n}(\mathbb{C})$. The cohomology group $H^{\bullet}\left(\mathcal{B}_{\mu}\right)$ of $\mathcal{B}_{\mu}$ is naturally an $S_{n}$-module, and the induced $\mathcal{H}_{n}$-module $\operatorname{ind}_{\mathbb{C S} S_{n}}^{\mathcal{H}_{n}} H^{\bullet}\left(\mathcal{B}_{\mu}\right) \cong \mathcal{C}_{n} \otimes H^{\bullet}\left(\mathcal{B}_{\mu}\right)$ is $\mathbb{Z}_{+}$-graded, with the grading inherited from the one on $H^{\bullet}\left(\mathcal{B}_{\mu}\right)$. Define a polynomial $C_{\xi \mu}^{-}(t)$ (as a graded multiplicity) by

$$
C_{\xi \mu}^{-}(t):=\sum_{i \geq 0} t^{i}\left(\operatorname{dim} \operatorname{Hom}_{\mathcal{H}_{n}}\left(D^{\xi}, \mathcal{C}_{n} \otimes H^{2 i}\left(\mathcal{B}_{\mu}\right)\right)\right),
$$

which should be morally viewed as a version of Springer theory (undeveloped yet) of the queer Lie supergroups.

The queer Lie superalgebra $\mathfrak{q}(n)$ contains the general linear Lie algebra $\mathfrak{g l}(n)$ as its even subalgebra, and its irreducible polynomial representations $V(\xi)$ are parameterized by highest weights $\xi \in \mathcal{S} \mathcal{P}$ with $\ell(\xi) \leq n$. Let $e$ be a regular nilpotent element in $\mathfrak{g l}(n)$, regarded as an element in the even subalgebra of $\mathfrak{q}(n)$. For $\mu \in \mathcal{P}$ with $\ell(\mu) \leq n$, using the action of $e$ we introduce a Brylinski-Kostant filtration on the weight space $V(\xi)_{\mu}$ and denote by $\gamma_{\xi \mu}^{-}(t)$ the associated polynomial (or $q$-weight multiplicity).

The spin Kostka polynomial $K_{\xi \mu}^{-}(t)$ can be interpreted in terms of graded multiplicity $C_{\xi \mu}^{-}(t)$ as well as the $q$-weight multiplicity $\gamma_{\xi \mu}^{-}(t)$ as follows (also see Proposition 3.3 for another expression of $q$-weight multiplicity). 
Theorem B Suppose $\xi \in \mathcal{S P}_{n}, \mu \in \mathcal{P}_{n}$. Then we have

(1) $K_{\xi \mu}^{-}(t)=2^{\frac{\ell(\xi)-\delta(\xi)}{2}} C_{\xi \mu}^{-}\left(t^{-1}\right) t^{n(\mu)}$.

(2) $K_{\xi \mu}^{-}(t)=2^{\frac{\ell(\xi)-\delta(\xi)}{2}} \gamma_{\xi \mu}^{-}(t)$.

Theorem $\mathrm{A}(6)$ and Theorem $\mathrm{B}(1)$ for $\mu=\left(1^{n}\right)$ (note that $\mathcal{B}_{\left(1^{n}\right)}=\mathcal{B}$ ) are reinterpretation of a main result of our previous work [21] on the spin coinvariant algebra. Actually, this has been our original motivation of introducing spin Kostka polynomials and finding representation theoretic interpretations. The two interpretations of the spin Kostka polynomials in Theorem B are connected to each other via SchurSergeev duality between $\mathfrak{q}(n)$ and the Hecke-Clifford algebra [16].

\section{4}

In Sect. 4, we construct a map $\Phi$ and a commutative diagram:

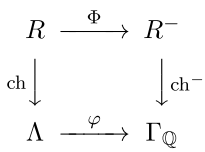

where $\varphi$ given in (4.1) is as in [13, III, Sect. 8, Example 10], and ch and $\mathrm{ch}^{-}$are characteristic maps from the module categories of $S_{n}$ and $\mathcal{H}_{n}$, respectively. The commutative diagram serves as a bridge of various old and new constructions, and the use of Hecke-Clifford algebra provides simple representation theoretic interpretations of some symmetric function results in [19] and [13]. We further define a spin analogue $H_{\mu}^{-}(x ; t)$ of the normalized Hall-Littlewood function $H_{\mu}(x ; t)$ via the spin Kostka polynomials. We show that $H_{\mu}^{-}(x ; t)$ coincides with the image of $H_{\mu}(x ; t)$ under the map $\varphi$, and it satisfies additional favorable properties (see Theorem 4.4).

We also sketch a similar construction of the spin Macdonald polynomials $H_{\mu}^{-}(x ; q, t)$ and the spin $q, t$-Kostka polynomials $K_{\xi \mu}^{-}(q, t)$. The use of $\Phi$ and $\varphi$ makes such a $q, t$-generalization possible.

\section{5}

The paper is organized as follows. In Sect. 2, we review some basics on Kostka polynomials, introduce the spin Kostka polynomials, and then prove Theorem A. The representation theoretic interpretations of spin Kostka polynomials are presented and Theorem B is proved in Sect. 3. In Sect. 4, we introduce the spin Hall-Littlewood functions, spin Macdonald polynomials and spin $q, t$-Kostka polynomials. We end the paper with a list of open problems.

\section{Spin Kostka polynomials}

In this section, we shall first review the basics for Kostka polynomials. Then, we introduce the spin Kostka polynomials and prove that these polynomials satisfy the properties listed in Theorem A. 


\subsection{Basics on Kostka polynomials}

A partition $\lambda$ will be identified with its Young diagram, that is, $\lambda=\left\{(i, j) \in \mathbb{Z}^{2} \mid 1 \leq\right.$ $\left.i \leq \ell(\lambda), 1 \leq j \leq \lambda_{i}\right\}$. To each cell $(i, j) \in \lambda$, we associate its content $c_{i j}=j-i$ and hook length $h_{i j}=\lambda_{i}+\lambda_{j}^{\prime}-i-j+1$, where $\lambda^{\prime}=\left(\lambda_{1}^{\prime}, \lambda_{2}^{\prime}, \ldots\right)$ is the conjugate partition of $\lambda$. For $\lambda, \mu \in \mathcal{P}$, let $K_{\lambda \mu}$ be the Kostka number, which counts the number of semistandard tableaux of shape $\lambda$ and weight $\mu$. We write $|\lambda|=n$ for $\lambda \in \mathcal{P}_{n}$. The dominance order on $\mathcal{P}$ is defined by letting

$$
\lambda \geq \mu \quad \Leftrightarrow \quad|\lambda|=|\mu| \quad \text { and } \quad \lambda_{1}+\cdots+\lambda_{i} \geq \mu_{1}+\cdots+\mu_{i}, \quad \forall i \geq 1 .
$$

Let $\lambda, \mu \in \mathcal{P}$. The Koksta polynomial $K_{\lambda \mu}(t)$ is defined by

$$
s_{\lambda}(x)=\sum_{\mu} K_{\lambda \mu}(t) P_{\mu}(x ; t),
$$

where $P_{\mu}(x ; t)$ and $s_{\lambda}(x)$ are Hall-Littlewood functions and Schur functions, respectively (cf. [13, III, Sect. 2]). The following is a summary of a long development by many authors.

Theorem 2.1 (Cf. [13], III, Sect. 6) Suppose $\lambda, \mu \in \mathcal{P}_{n}$. Then the Kostka polynomial $K_{\lambda \mu}(t)$ satisfies the following properties:

(1) $K_{\lambda \mu}(t)=0$ unless $\lambda \geq \mu ; K_{\lambda \lambda}(t)=1$.

(2) The degree of $K_{\lambda \mu}(t)$ is $n(\mu)-n(\lambda)$.

(3) $K_{\lambda \mu}(t)$ is a polynomial with non-negative integer coefficients.

(4) $K_{\lambda \mu}(1)=K_{\lambda \mu}$.

(5) $K_{(n) \mu}(t)=t^{n(\mu)}$.

(6) $K_{\lambda\left(1^{n}\right)}=\frac{t^{n\left(\lambda^{\prime}\right)}(1-t)\left(1-t^{2}\right) \cdots\left(1-t^{n}\right)}{\prod_{(i, j) \in \lambda}\left(1-t^{h_{i j}}\right)}$.

Let $\mathcal{B}$ be the flag variety for the general linear group $G L_{n}(\mathbb{C})$. For a partition $\mu$ of $n$, let $\mathcal{B}_{\mu}$ denote the subvariety of $\mathcal{B}$ consisting of flags preserved by the Jordan canonical form $J_{\mu}$ of shape $\mu$. It is known [18] that the cohomology group $H^{\bullet}\left(\mathcal{B}_{\mu}\right)$ of $\mathcal{B}_{\mu}$ with complex coefficient affords a graded representation of the symmetric group $S_{n}$. Define $C_{\lambda \mu}(t)$ by

$$
C_{\lambda \mu}(t)=\sum_{i \geq 0} t^{i} \operatorname{Hom}_{S_{n}}\left(S^{\lambda}, H^{2 i}\left(\mathcal{B}_{\mu}\right)\right),
$$

where $S^{\lambda}$ denotes the Specht module over $S_{n}$.

Theorem 2.2 (Cf. [13], III, Sect. 7, Example 8; [5], (5.7)) The following holds for $\lambda, \mu \in \mathcal{P}$ :

$$
K_{\lambda \mu}(t)=C_{\lambda \mu}\left(t^{-1}\right) t^{n(\mu)}
$$


It is well known that the cohomology $\operatorname{ring} H^{\bullet}(\mathcal{B})$ of the flag variety $\mathcal{B}$ coincides with the coinvariant algebra of the symmetric group $S_{n}$. Garsia and Procesi [5] gave a purely algebraic construction of the graded $S_{n}$-module $H^{\bullet}\left(\mathcal{B}_{\mu}\right)$ in terms of quotients of the coinvariant algebra of symmetric groups as well as a proof of Theorem 2.2.

Denote by $\left\{\epsilon_{1}, \ldots, \epsilon_{n}\right\}$ the basis dual to the standard basis $\left\{E_{i i} \mid 1 \leq i \leq n\right\}$ in the standard Cartan subalgebra of $\mathfrak{g l}(n)$, where $E_{i i}$ denotes the matrix whose $(i, i)$ th entry is 1 and zero elsewhere. Let $L(\lambda)$ be the irreducible $\mathfrak{g l}(n)$-module with highest weight $\lambda$ for $\lambda \in \mathcal{P}$ with $\ell(\lambda) \leq n$. For each $\mu \in \mathcal{P}$ with $\ell(\mu) \leq n$, define the $q$-weight multiplicity of weight $\mu$ in $L(\lambda)$ to be

$$
m_{\mu}^{\lambda}(t)=\left[e^{\mu}\right] \frac{\prod_{\alpha>0}\left(1-e^{-\alpha}\right)}{\prod_{\alpha>0}\left(1-t e^{-\alpha}\right)} \operatorname{ch} L(\lambda),
$$

where the product $\prod_{\alpha>0}$ is over all positive roots $\left\{\epsilon_{i}-\epsilon_{j} \mid 1 \leq i<j \leq n\right\}$ for $\mathfrak{g l}(n)$ and $\left[e^{\mu}\right] f\left(e^{\epsilon_{1}}, \ldots, e^{\epsilon_{n}}\right)$ denotes the coefficient of the monomial $e^{\mu}$ in a formal series $f\left(e^{\epsilon_{1}}, \ldots, e^{\epsilon_{n}}\right)$. According to a conjecture of Lusztig proved by Kato [9, 12], we have

$$
K_{\lambda \mu}(t)=m_{\mu}^{\lambda}(t) .
$$

Let $e$ be a regular nilpotent element in the Lie algebra $\mathfrak{g l}(n)$. For each $\mu \in \mathcal{P}$ with $\ell(\mu) \leq n$, define the Brylinski-Kostant filtration on the weight space $L(\lambda)_{\mu}$ by

$$
0 \subseteq J_{e}^{0}\left(L(\lambda)_{\mu}\right) \subseteq J_{e}^{1}\left(L(\lambda)_{\mu}\right) \subseteq \cdots
$$

with

$$
J_{e}^{k}\left(L(\lambda)_{\mu}\right)=\left\{v \in L(\lambda)_{\mu} \mid e^{k+1} v=0\right\},
$$

where we assume $J_{e}^{-1}\left(L(\lambda)_{\mu}\right)=\{0\}$. Define a polynomial $\gamma_{\lambda \mu}(t)$ by

$$
\gamma_{\lambda \mu}(t)=\sum_{k \geq 0}\left(\operatorname{dim} J_{e}^{k}\left(L(\lambda)_{\mu}\right) / J_{e}^{k-1}\left(L(\lambda)_{\mu}\right)\right) t^{k} .
$$

The following theorem is due to R. Brylinski (see [1, Theorem 3.4] and (2.3)).

Theorem 2.3 Suppose $\lambda, \mu \in \mathcal{P}$ with $\ell(\lambda) \leq n$ and $\ell(\mu) \leq n$. Then we have

$$
K_{\lambda \mu}(t)=\gamma_{\lambda \mu}(t)
$$

\subsection{Schur $Q$-functions and spin Kostka polynomials}

Given a partition $\lambda \in \mathcal{P}$, suppose that the main diagonal of the Young diagram $\lambda$ contains $r$ cells. Let $\alpha_{i}=\lambda_{i}-i$ be the number of cells in the $i$ th row of $\lambda$ strictly to the right of $(i, i)$, and let $\beta_{i}=\lambda_{i}^{\prime}-i$ be the number of cells in the $i$ th column of $\lambda$ strictly below $(i, i)$, for $1 \leq i \leq r$. We have $\alpha_{1}>\alpha_{2}>\cdots>\alpha_{r} \geq 0$ and $\beta_{1}>\beta_{2}>\cdots>$ $\beta_{r} \geq 0$. Then the Frobenius notation for a partition is $\lambda=\left(\alpha_{1}, \ldots, \alpha_{r} \mid \beta_{1}, \ldots, \beta_{r}\right)$. For example, if $\lambda=(5,4,3,1)$, then $\alpha=(4,2,0), \beta=(3,1,0)$ and hence $\lambda=$ $(4,2,0 \mid 3,1,0)$ in Frobenius notation. 
For a strict partition $\xi \in \mathcal{S P}_{n}$, let $\xi^{*}$ be the associated shifted Young diagram, that is,

$$
\xi^{*}=\left\{(i, j) \mid 1 \leq i \leq \ell(\xi), i \leq j \leq \xi_{i}+i-1\right\}
$$

which is obtained from the ordinary Young diagram by shifting the $k$ th row to the right by $k-1$ squares, for each $k$. Given $\xi \in S \mathcal{P}_{n}$ with $\ell(\xi)=\ell$, define its double partition (or double diagram) $\widetilde{\xi}$ to be $\widetilde{\xi}=\left(\xi_{1}, \ldots, \xi_{\ell} \mid \xi_{1}-1, \xi_{2}-1, \ldots, \xi_{\ell}-1\right)$ in Frobenius notation. Clearly, the shifted Young diagram $\xi^{*}$ coincides with the part of $\widetilde{\xi}$ that lies above the main diagonal. For each cell $(i, j) \in \xi^{*}$, denote by $h_{i j}^{*}$ the associated hook length in the Young diagram $\widetilde{\xi}$, and set the content $c_{i j}=j-i$.

For example, let $\xi=(4,2,1)$. The corresponding shifted diagram and double diagram are
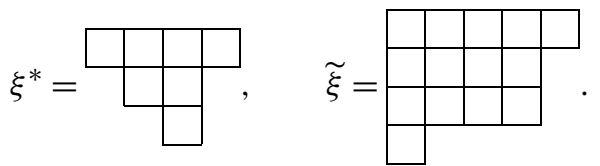

The contents of $\xi$ are listed in the corresponding cells of $\xi^{*}$ as follows:

$$
\begin{array}{|l|l|l|l|}
\hline 0 & 1 & 2 & 3 \\
\hline & 0 & 1 & \\
\cline { 1 - 2 } & & 0 & \multicolumn{1}{|c}{} \\
\cline { 2 - 3 } & &
\end{array}
$$

The shifted hook lengths for each cell in $\xi^{*}$ are defined as the usual hook lengths for the corresponding cell in the double diagram $\widetilde{\xi}$, as follows:
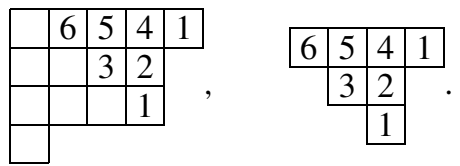

Denote by $\mathbf{P}^{\prime}$ the ordered alphabet $\left\{1^{\prime}<1<2^{\prime}<2<3^{\prime}<3 \cdots\right\}$. The symbols $1^{\prime}, 2^{\prime}, 3^{\prime}, \ldots$ are said to be marked, and we shall denote by $|a|$ the unmarked version of any $a \in \mathbf{P}^{\prime}$; that is, $\left|k^{\prime}\right|=|k|=k$ for each $k \in \mathbb{N}$. For a strict partition $\xi$, a marked shifted tableau $T$ of shape $\xi$, or a marked shifted $\xi$-tableau $T$, is an assignment $T: \xi^{*} \rightarrow \mathbf{P}^{\prime}$ satisfying:

(M1) The letters are weakly increasing along each row and column.

(M2) The letters $\{1,2,3, \ldots\}$ are strictly increasing along each column.

(M3) The letters $\left\{1^{\prime}, 2^{\prime}, 3^{\prime}, \ldots\right\}$ are strictly increasing along each row.

For a marked shifted tableau $T$ of shape $\xi$, let $\alpha_{k}$ be the number of cells $(i, j) \in \xi^{*}$ such that $|T(i, j)|=k$ for $k \geq 1$. The sequence $\left(\alpha_{1}, \alpha_{2}, \alpha_{3}, \ldots\right)$ is called the weight of $T$. The Schur $Q$-function associated to $\xi$ can be interpreted as (see $[13,14,19]$ )

$$
Q_{\xi}(x)=\sum_{T} x^{T}
$$


where the summation is taken over all marked shifted tableaux of shape $\xi$, and $x^{T}=$ $x_{1}^{\alpha_{1}} x_{2}^{\alpha_{2}} x_{3}^{\alpha_{3}} \ldots$ if $T$ has weight $\left(\alpha_{1}, \alpha_{2}, \alpha_{3}, \ldots\right)$. Set

$$
K_{\xi \mu}^{-}=\#\{T \mid T \text { is a marked shifted tableau of shape } \xi \text { and weight } \mu\} .
$$

Then we have

$$
Q_{\xi}(x)=\sum_{\mu} K_{\xi \mu}^{-} m_{\mu}(x) .
$$

It will be convenient to introduce another family of symmetric functions $q_{\lambda}(x)$ for any partition $\lambda=\left(\lambda_{1}, \lambda_{2}, \ldots\right)$ as follows: $q_{0}(x)=1, q_{r}(x)=Q_{(r)}(x)$ for $r \geq 1$, and $q_{\lambda}(x)=q_{\lambda_{1}}(x) q_{\lambda_{2}}(x) \cdots$. The generating function $Q(u)$ for $q_{r}(x)$ is

$$
\sum_{r \geq 0} q_{r}(x) u^{r}=Q(u)=\prod_{i} \frac{1+x_{i} u}{1-x_{i} u} .
$$

We will write $q_{r}=q_{r}(x)$, etc., whenever there is no need to specify the variables. Let $\Gamma$ be the $\mathbb{Z}$-algebra generated by $q_{r}, r \geq 1$, that is,

$$
\Gamma=\mathbb{Z}\left[q_{1}, q_{2}, \ldots\right] \text {. }
$$

It is known that the set $\left\{Q_{\xi} \mid \xi \in \mathcal{S P}\right\}$ forms a $\mathbb{Z}$-basis of $\Gamma$.

Definition 2.4 The spin Kostka polynomials $K_{\xi \mu}^{-}(t)$ for $\xi \in \mathcal{S P}$ and $\mu \in \mathcal{P}$ are given by

$$
Q_{\xi}(x)=\sum_{\mu} K_{\xi \mu}^{-}(t) P_{\mu}(x ; t) .
$$

2.3 Properties of spin Kostka polynomials

For $\xi \in \mathcal{S P}$, write

$$
Q_{\xi}(x)=\sum_{\lambda \in \mathcal{P}} b_{\xi \lambda} s_{\lambda}(x),
$$

for some suitable constants $b \xi \lambda$.

Proposition 2.5 The following holds for $\xi \in \mathcal{S P}$ and $\mu \in \mathcal{P}$ :

$$
K_{\xi \mu}^{-}(t)=\sum_{\lambda \in \mathcal{P}} b_{\xi \lambda} K_{\lambda \mu}(t)
$$

Proof By (2.1) and (2.8), one can deduce that

$$
\sum_{\mu} K_{\xi \mu}^{-}(t) P_{\mu}(x ; t)=\sum_{\lambda, \mu} b_{\xi \lambda} K_{\lambda \mu}(t) P_{\mu}(x ; t) .
$$

The proposition now follows from the fact that the Hall-Littlewood functions $P_{\mu}(x ; t)$ are linearly independent in $\mathbb{Z}[t] \otimes_{\mathbb{Z}} \Lambda$. 
The usual Kostka polynomial satisfies that $K_{\lambda \mu}(0)=\delta_{\lambda \mu}$. It follows from Proposition 2.5 that

$$
K_{\xi \mu}^{-}(0)=b_{\xi \mu}
$$

For $\xi \in \mathcal{S P}, \lambda \in \mathcal{P}$, set

$$
g_{\xi \lambda}=2^{-\ell(\xi)} b_{\xi \lambda}
$$

Lemma 2.6 ([19], Theorem 9.3; [13], III (8.17)) The following holds for $\xi \in \mathcal{S P}, \lambda \in \mathcal{P}$ :

$$
g_{\xi \lambda} \in \mathbb{Z}_{+} ; \quad g_{\xi \lambda}=0 \quad \text { unless } \xi \geq \lambda ; \quad g_{\xi \xi}=1 .
$$

Stembridge [19] proved Lemma 2.6 by giving a combinatorial formula for $g_{\xi \lambda}$ in terms of marked shifted tableaux. We shall give a simple representation theoretic proof of Lemma 2.6 in Sect. 3.4 for the sake of completeness.

Proof of Theorem A Combining Theorem 2.1(1)-(3), Lemma 2.6 and Proposition 2.5 , we can easily verify that the spin Kostka polynomial $K_{\xi \mu}^{-}(t)$ must satisfy the properties (1)-(3) in Theorem A.

It is known that $P_{\mu}(x ; 1)=m_{\mu}$ and hence by (2.4) we have $K_{\xi \mu}^{-}(1)=K_{\xi \mu}^{-}$. Also, $Q_{\xi}=2^{\ell(\xi)} P_{\xi}(x ;-1)$, and $\left\{P_{\mu}(x ;-1) \mid \mu \in \mathcal{P}\right\}$ forms a basis for $\Lambda$ (see [13, p. 253]). Hence (4) is proved.

By [13, III, Sect. 3, Example 1(3)] we have

$$
\prod_{i \geq 1} \frac{1+x_{i}}{1-x_{i}}=\sum_{\mu} t^{n(\mu)} \prod_{j=1}^{\ell(\mu)}\left(1+t^{1-j}\right) P_{\mu}(x ; t) .
$$

Comparing the degree $n$ terms of (2.11) and (2.5), we obtain

$$
Q_{(n)}(x)=q_{n}(x)=\sum_{\mu \in \mathcal{P}_{n}} t^{n(\mu)} \prod_{j=1}^{\ell(\mu)}\left(1+t^{1-j}\right) P_{\mu}(x ; t) .
$$

Hence (5) is proved.

Part (6) actually follows from Theorem $\mathrm{B}(1)$ and the main result of [21], and let us postpone its proof after completing the proof of Theorem $\mathrm{B}(1)$.

\section{Spin Kostka polynomials and representation theory}

In this section, we shall give two interpretations of spin Kostka polynomials in representation theory.

3.1 The Frobenius characteristic map ch

Denote by $S_{n}$-mod the category of finite-dimensional $S_{n}$-modules. Let $R_{n}=$ $K\left(S_{n}\right.$-mod $)$ be Grothendieck group of the category $S_{n}$-mod and set 


$$
R=\bigoplus_{n \geq 0} R_{n}
$$

Recall that $R_{n}$ admits an inner product by declaring the irreducible characters to be orthonormal. Also there exists an inner product (, ) on the ring $\Lambda$ such that the Schur functions $s_{\lambda}$ form an orthonormal basis. The Frobenius characteristic map ch : $R \rightarrow$ $\Lambda$ preserves the inner products and it satisfies that

$$
\begin{aligned}
\operatorname{ch}\left(\left[S^{\lambda}\right]\right) & =s_{\lambda}, \\
\operatorname{ch}\left(\operatorname{ind}_{\mathbb{C} S_{\lambda}}^{\mathbb{C} S_{n}} \mathbf{1}\right) & =h_{\lambda}, \quad \lambda \in \mathcal{P}_{n},
\end{aligned}
$$

where 1 denotes the trivial character.

3.2 Hecke-Clifford algebra $\mathcal{H}_{n}$ and the characteristic map $\mathrm{ch}^{-}$

A superalgebra $A=A_{\overline{0}} \oplus A_{\overline{1}}$ satisfies $A_{i} \cdot A_{j} \subseteq A_{i+j}$ for $i, j \in \mathbb{Z}_{2}$. Denote by $\mathcal{C}_{n}$ the Clifford superalgebra generated by the odd elements $c_{1}, \ldots, c_{n}$, subject to the relations $c_{i}^{2}=1, c_{i} c_{j}=-c_{j} c_{i}$ for $1 \leq i \neq j \leq n$. The symmetric group $S_{n}$ acts as automorphisms on the Clifford algebra $\mathcal{C}_{n}$ by permuting its generators, and the Hecke-Clifford algebra is defined to be the semi-direct product $\mathcal{H}_{n}=\mathcal{C}_{n} \rtimes \mathbb{C} S_{n}$ with $\sigma c_{i}=c_{\sigma(i)} \sigma$, for $\sigma \in S_{n}, 1 \leq i \leq n$. Note that the algebra $\mathcal{H}_{n}$ is naturally a superalgebra by letting each $\sigma \in S_{n}$ be even and each $c_{i}$ be odd.

A module over a superalgebra, e.g. $\mathcal{H}_{n}$, is always understood to be $\mathbb{Z}_{2}$-graded in this paper. It is known $[8,16,19]$ (cf. [10]) that there exists an irreducible $\mathcal{H}_{n}$-module $D^{\xi}$ for each strict partition $\xi \in \mathcal{S} \mathcal{P}_{n}$ and $\left\{D^{\xi} \mid \xi \in \mathcal{S} \mathcal{P}_{n}\right\}$ forms a complete set of non-isomorphic irreducible $\mathcal{H}_{n}$-modules. A superalgebra analogue of Schur's lemma states that the endomorphism algebra of a finite-dimensional irreducible module over a superalgebra is either one-dimensional or two-dimensional. It turns out that $[8,16]$

$$
\operatorname{dim} \operatorname{Hom}_{\mathcal{H}_{n}}\left(D^{\xi}, D^{\xi}\right)=2^{\delta(\xi)} .
$$

Denote by $\mathcal{H}_{n}$-smod the category of finite-dimensional $\mathcal{H}_{n}$-supermodules. Let $R_{n}^{-}$ be the Grothendieck group of the category $\mathcal{H}_{n}$-smod and define

$$
R^{-}=\bigoplus_{n \geq 0} R_{n}^{-}, \quad R_{\mathbb{Q}}^{-}=\mathbb{Q} \otimes_{\mathbb{Z}} R^{-} .
$$

Recall the ring $\Gamma$ from (2.6) and set $\Gamma_{\mathbb{Q}}=\mathbb{Q} \otimes_{\mathbb{Z}} \Gamma$. As a spin analogue of the Frobenius characteristic map ch, there exists an isomorphism of graded vector spaces [8]

$$
\begin{aligned}
\mathrm{ch}^{-}: R_{\mathbb{Q}}^{-} & \longrightarrow \Gamma_{\mathbb{Q}} \\
{\left[D^{\xi}\right] } & \mapsto 2^{-\frac{\ell(\xi)-\delta(\xi)}{2}} Q_{\xi}, \\
\operatorname{ind}_{\mathbb{C} S_{\mu}}^{\mathcal{H}} \mathbf{1} & \mapsto q_{\mu} .
\end{aligned}
$$

It is useful to note that $\mathrm{ch}^{-}$is related to $\mathrm{ch}$ as follows:

$$
\operatorname{ch}^{-}(\zeta)=\operatorname{ch}\left(\operatorname{res}_{\mathbb{C} S_{n}}^{\mathcal{H}_{n}} \zeta\right), \quad \text { for } \zeta \in R_{n}^{-}
$$




\subsection{Spin Kostka polynomials and graded multiplicity}

Up to some 2-power, $g_{\xi \lambda}$ has the following representation theoretic interpretation.

Lemma 3.1 Suppose $\xi \in \mathcal{S P}_{n}, \lambda \in \mathcal{P}_{n}$. The following holds:

$$
\operatorname{dim} \operatorname{Hom}_{\mathcal{H}_{n}}\left(D^{\xi}, \operatorname{ind}_{\mathbb{C} S_{n}}^{\mathcal{H}_{n}} S^{\lambda}\right)=2^{\frac{\ell(\xi)+\delta(\xi)}{2}} g_{\xi \lambda}
$$

Proof Since the $\mathcal{H}_{n}$-module $\operatorname{ind}_{\mathbb{C} S_{n}}^{\mathcal{H}_{n}} S^{\lambda}$ is semisimple, we have

$$
\begin{aligned}
\operatorname{dim} \operatorname{Hom}_{\mathcal{H}_{n}}\left(D^{\xi}, \operatorname{ind}_{\mathbb{C S}_{n}}^{\mathcal{H}_{n}} S^{\lambda}\right) & =\operatorname{dim}_{H_{\mathcal{H}}}\left(\operatorname{ind}_{\mathbb{C} S_{n}}^{\mathcal{H}_{n}} S^{\lambda}, D^{\xi}\right) \\
& =\operatorname{dim}_{\operatorname{Hom}_{\mathbb{C} S_{n}}}\left(S^{\lambda}, \operatorname{res}_{\mathbb{C} S_{n}}^{\mathcal{H} D_{n}}\right) \\
& =\left(s_{\lambda}, \operatorname{ch}\left(\operatorname{res}_{\mathbb{C} S_{n}}^{\mathcal{H}_{n}} D^{\xi}\right)\right) \\
& =\left(s_{\lambda}, \operatorname{ch}^{-}\left(D^{\xi}\right)\right) \\
& =\left(s_{\lambda}, 2^{-\frac{\ell(\xi)-\delta(\xi)}{2}} Q_{\xi}(x)\right) \\
& =2^{\frac{\ell(\xi)+\delta(\xi)}{2}} g_{\xi \lambda},
\end{aligned}
$$

where the second equation uses the Frobenius reciprocity, the third equation uses the fact that ch is an isometry, the fourth, fifth and sixth equations follow from (3.6), (3.4), and (2.8), respectively.

Proof of Theorem $B(1)$ Suppose $\xi \in S \mathcal{P}_{n}$ and $\mu \in \mathcal{P}_{n}$. By Proposition 2.5 and Theorem 2.2, we obtain

$$
K_{\xi \mu}^{-}(t)=\sum_{\lambda \in \mathcal{P}_{n}} b_{\xi \lambda} K_{\lambda \mu}(t)=\sum_{\lambda \in \mathcal{P}_{n}} b_{\xi \lambda} C_{\lambda \mu}\left(t^{-1}\right) t^{n(\mu)} .
$$

On the other hand, recalling the definition of $C_{\xi \mu}^{-}(t)$ from (1.1) and the definition of $C_{\lambda \mu}(t)$ from (2.2), we have by Lemma 3.1 that

$$
\begin{aligned}
C_{\xi \mu}^{-}(t) & =\sum_{i \geq 0} t^{i}\left(\operatorname{dim}^{\operatorname{Hom}_{\mathcal{H}_{n}}}\left(D^{\xi}, \operatorname{ind}_{\mathbb{C} S_{n}}^{\mathcal{H}_{n}} H^{2 i}\left(\mathcal{B}_{\mu}\right)\right)\right) \\
& =\sum_{\lambda} C_{\lambda \mu}(t) \operatorname{dim} \operatorname{Hom}_{\mathcal{H}_{n}}\left(D^{\xi}, \operatorname{ind}_{\mathbb{C} S_{n}}^{\mathcal{H}_{n}} S^{\lambda}\right) \\
& =2^{-\frac{\ell(\xi)-\delta(\xi)}{2}} \sum_{\lambda \in \mathcal{P}_{n}} b_{\xi \lambda} C_{\lambda \mu}(t) .
\end{aligned}
$$

Now Theorem B(1) follows by comparing the above two identities.

With Theorem B(1) at hand, we can complete the proof of Theorem A. 
Proof of Theorem $A(6)$ Suppose $\xi \in S \mathcal{P}_{n}$. Observe that $\mathcal{B}_{\left(1^{n}\right)}=\mathcal{B}$ and it is well known that $H^{\bullet}(\mathcal{B})$ is isomorphic to the coinvariant algebra of the symmetric group $S_{n}$. Hence by [21, Theorem 3.5] and (3.3) we obtain

$$
C_{\xi\left(1^{n}\right)}^{-}(t)=2^{-\frac{\ell(\xi)-\delta(\xi)}{2}} \frac{t^{n(\xi)}(1-t)\left(1-t^{2}\right) \cdots\left(1-t^{n}\right) \prod_{(i, j) \in \xi^{*}}\left(1+t^{c_{i j}}\right)}{\prod_{(i, j) \in \xi^{*}}\left(1-t^{h_{i j}^{*}}\right)},
$$

where $\xi^{*}$ is the shifted Young diagram associated to $\xi$ and $c_{i j}, h_{i j}^{*}$ are contents and shifted hook lengths for a cell $(i, j) \in \xi$, respectively. This together with Theorem $\mathrm{B}(1)$ gives rise to

$$
\begin{aligned}
K_{\xi\left(1^{n}\right)}^{-}(t) & =\frac{t^{\frac{n(n-1)}{2}-n(\xi)}\left(1-t^{-1}\right)\left(1-t^{-2}\right) \cdots\left(1-t^{-n}\right) \prod_{(i, j) \in \xi^{*}}\left(1+t^{-c_{i j}}\right)}{\prod_{(i, j) \in \xi^{*}}\left(1-t^{-h_{i j}^{*}}\right)} \\
& =\frac{t^{-n-n(\xi)+\sum_{(i, j) \in \xi^{*}} h_{i j}^{*}}(1-t)\left(1-t^{2}\right) \cdots\left(1-t^{n}\right) \prod_{(i, j) \in \xi^{*}}\left(1+t^{c_{i j}}\right)}{t^{\sum_{(i, j) \in \xi^{*}} c_{i j}} \prod_{(i, j) \in \xi^{*}}\left(1-t^{h_{i j}^{*}}\right)} \\
& =\frac{t^{n(\xi)}(1-t)\left(1-t^{2}\right) \cdots\left(1-t^{n}\right) \prod_{(i, j) \in \xi^{*}}\left(1+t^{c_{i j}}\right)}{\prod_{(i, j) \in \xi^{*}}\left(1-t^{h_{i j}^{*}}\right)},
\end{aligned}
$$

where the last equality can be derived by noting that the contents $c_{i j}$ are $0,1, \ldots, \xi_{i}-$ 1 and the fact (cf. [13, III, Sect. 8, Example 12]) that in the $i$ th row of $\xi^{*}$, the hook lengths $h_{i j}^{*}$ for $i \leq j \leq \xi_{i}+i-1$ are $1,2, \ldots, \xi_{i}, \xi_{i}+\xi_{i+1}, \xi_{i}+\xi_{i+2}, \ldots, \xi_{i}+\xi_{\ell}$ with exception $\xi_{i}-\xi_{i+1}, \xi_{i}-\xi_{i+2}, \ldots, \xi_{i}-\xi_{\ell}$.

\subsection{Spin Kostka polynomials and $q$-weight multiplicity}

The queer Lie superalgebra, denoted by $\mathfrak{q}(n)$, can be viewed as the subalgebra of the general linear Lie superalgebra $\mathfrak{g l}(n \mid n)$ consisting of matrices of the form

$$
\left(\begin{array}{ll}
a & b \\
b & a
\end{array}\right)
$$

where $a$ and $b$ are arbitrary $n \times n$ matrices. Let $\mathfrak{g}=\mathfrak{q}(n)$ and $I(n \mid n)=\{\overline{1}, \ldots, \bar{n}$, $1, \ldots, n\}$. The even (respectively, odd) part $\mathfrak{g}_{\overline{0}}$ (respectively, $\mathfrak{g}_{\overline{1}}$ ) consists of those matrices of the form (3.7) with $b=0$ (respectively, $a=0$ ). Denote by $E_{i j}$ for $i, j \in I(n \mid n)$ the standard elementary matrix with the $(i, j)$ th entry being 1 and zero elsewhere. Fix the triangular decomposition

$$
\mathfrak{g}=\mathfrak{n}^{-} \oplus \mathfrak{h} \oplus \mathfrak{n}^{+}
$$

where $\mathfrak{h}$ (respectively, $\mathfrak{n}^{+}, \mathfrak{n}^{-}$) is the subalgebra of $\mathfrak{g}$ which consists of matrices of the form (3.7) with $a, b$ being arbitrary diagonal (respectively, upper triangular, lower triangluar) matrices. Let $\mathfrak{b}=\mathfrak{h} \oplus \mathfrak{n}^{+}$.

Let $\left\{\epsilon_{i} \mid i=1, \ldots, n\right\}$ be the basis dual to the standard basis $\left\{E_{i i}+E_{\bar{i}, \bar{i}} \mid i=\right.$ $1, \ldots, n\}$ for the even subalgebra $\mathfrak{h}_{\overline{0}}$ of $\mathfrak{h}$, where $\mathfrak{h}_{\overline{0}}$ is identified with the standard 
Cartan subalgebra of $\mathfrak{g l}(n)$ via the natural isomorphism $\mathfrak{q}(n)_{\overline{0}} \cong \mathfrak{g l}(n)$. With respect to $\mathfrak{h}_{\overline{0}}$ we have the root space decomposition $\mathfrak{g}=\mathfrak{h} \oplus \bigoplus_{\alpha \in \Delta} \mathfrak{g}_{\alpha}$ with roots $\Delta=$ $\left\{\epsilon_{i}-\epsilon_{j} \mid 1 \leq i \neq j \leq n\right\}$. The set of positive roots corresponding to the Borel subalgebra $\mathfrak{b}$ is

$$
\Delta^{+}=\left\{\epsilon_{i}-\epsilon_{j} \mid 1 \leq i<j \leq n\right\} .
$$

Noting that $\left[\mathfrak{h}_{\overline{1}}, \mathfrak{h}_{\overline{1}}\right]=\mathfrak{h}_{\overline{0}}$, the Lie superalgebra $\mathfrak{h}$ is not Abelian. For $\lambda \in$ $\sum_{i=1}^{n} \mathbb{Z} \epsilon_{i} \subset \mathfrak{h}_{\overline{0}}^{*}$, define the symmetric bilinear form $\langle\cdot, \cdot\rangle_{\lambda}$ on $\mathfrak{h}_{\overline{1}}$ by $\langle v, w\rangle_{\lambda}:=$ $\lambda([v, w])$. Let $\mathfrak{h}_{\overline{1}}^{\prime} \subseteq \mathfrak{h}_{\overline{1}}$ be a maximal isotropic subspace and consider the subalgebra $\mathfrak{h}^{\prime}=\mathfrak{h}_{\overline{0}} \oplus \mathfrak{h}_{\overline{1}}^{\prime}$. The one-dimensional $\mathfrak{h}_{0}$-module $\mathbb{C} v_{\lambda}$, defined by $h v_{\lambda}=\lambda(h) v_{\lambda}$, extends trivially to $\mathfrak{h}^{\prime}$. The induced $\mathfrak{h}$-module $W_{\lambda}:=\operatorname{Ind}_{\mathfrak{h}^{\prime}}^{\mathfrak{h}} \mathbb{C} v_{\lambda}$ is irreducible. Extend $W_{\lambda}$ to representation of $\mathfrak{b}$ by letting $\mathfrak{n}^{+} W_{\lambda}=0$. The induced $\mathfrak{g}$-module ind $\mathfrak{b}_{\mathfrak{b}}^{\mathfrak{g}} W_{\lambda}$ has a unique irreducible quotient, denoted by $V(\lambda)$. We have a weight space decomposition $V(\lambda)=\bigoplus_{\mu} V(\lambda)_{\mu}$, where a weight $\mu$ can be identified with a composition $\left(\mu_{1}, \ldots, \mu_{n}\right)$.

For $\xi \in \mathcal{S P}$ with $\ell(\xi) \leq n$, the $\mathfrak{q}(n)$-module $V(\xi)$ is finite-dimensional. Moreover, according to Sergeev [16], the character of $V(\xi)$ (by setting $x_{i}=e^{\epsilon_{i}}$ ) is

$$
\operatorname{ch} V(\xi)=2^{-\frac{\ell(\xi)-\delta(\xi)}{2}} Q_{\xi}\left(x_{1}, \ldots, x_{n}\right) .
$$

Regarding a regular nilpotent element $e$ in $\mathfrak{g l}(n)$ as an even element in $\mathfrak{q}(n)$, we define a Brylinski-Kostant filtration on the weight space $V(\xi)_{\mu}$ by

$$
0 \subseteq J_{e}^{0}\left(V(\xi)_{\mu}\right) \subseteq J_{e}^{1}\left(V(\xi)_{\mu}\right) \subseteq \cdots
$$

where

$$
J_{e}^{k}\left(V(\xi)_{\mu}\right):=\left\{v \in V(\xi)_{\mu} \mid e^{k+1} v=0\right\} .
$$

Define a polynomial $\gamma_{\xi \mu}^{-}(t)$ by

$$
\gamma_{\xi \mu}^{-}(t)=\sum_{k \geq 0}\left(\operatorname{dim} J_{e}^{k}\left(V(\xi)_{\mu}\right) / J_{e}^{k-1}\left(V(\xi)_{\mu}\right)\right) t^{k}
$$

Recall that $L(\lambda)$ denotes the irreducible representation of $\mathfrak{g l}(n)$ with highest weight $\lambda$ and recall $g_{\xi \lambda}$ from (2.9). Up to the same 2-power as in Lemma 3.1, $g_{\xi \lambda}$ has the following interpretation of branching coefficient.

Lemma 3.2 As a $\mathfrak{g l}(n)$-module, $V(\xi)$ can be decomposed as

$$
V(\xi) \cong \bigoplus_{\lambda \in \mathcal{P}, \ell(\lambda) \leq n} 2^{\frac{\ell(\xi)+\delta(\xi)}{2}} g_{\xi \lambda} L(\lambda) .
$$

Proof It suffices to verify on the character level. The corresponding character identity indeed follows from (2.8), (2.9), and (3.8), as the character of $L(\lambda)$ is given by the Schur function $s_{\lambda}$. 
Now we give a proof of Lemma 2.6 based on representation theory of $\mathfrak{q}(n)$ as promised. It is also possible to give another proof based on representation theory of Hecke-Clifford algebra $\mathcal{H}_{n}$.

Proof of Lemma 2.6 It follows by Lemma 3.2 that $g_{\xi \lambda} \geq 0$, and moreover, $g_{\xi \lambda}=0$ unless $\xi \geq \lambda$ (the dominance order for compositions coincide with the dominance order of weights for $\mathfrak{q}(n))$. The highest weight space for the $\mathfrak{q}(n)$-module $V(\xi)$ is $W_{\xi}$, which has dimension $2 \frac{\ell(\xi)+\delta(\xi)}{2}$. Hence, $g_{\xi \xi}=1$, by Lemma 3.2 again.

By (3.8), $2^{-\frac{\ell(\xi)+\delta(\xi)}{2}} \operatorname{ch} V(\xi)=2^{-\ell(\xi)} Q\left(x_{1}, \ldots, x_{n}\right)$, which is known to lie in $\Lambda$, cf. [13] (this fact can also be seen directly using representation theory of $\mathfrak{q}(n)$ ). Hence, $2^{-\ell(\xi)} Q\left(x_{1}, \ldots, x_{n}\right)$ is a $\mathbb{Z}$-linear combination of Schur polynomials $s_{\lambda}$. Combining with Lemma 3.2, this proves that $g_{\xi \lambda} \in \mathbb{Z}$.

We are ready to establish the Lie theoretic interpretation of spin Kostka polynomials.

Proof of Theorem $B(2)$ The Brylinski-Kostant filtration is defined via a regular nilpotent element in $\mathfrak{g l}(n) \cong \mathfrak{q}(n)_{\overline{0}}$, and thus it is compatible with the decomposition in Lemma 3.2. Hence, we have

$$
J_{e}^{k}\left(V(\xi)_{\mu}\right) \cong \bigoplus_{\lambda} 2^{\frac{\ell(\xi)+\delta(\xi)}{2}} g_{\xi \lambda} J_{e}^{k}\left(L(\lambda)_{\mu}\right) .
$$

It follows by the definitions of the polynomials $\gamma_{\xi \mu}^{-}(t)$ and $\gamma_{\lambda \mu}(t)$ that

$$
\gamma_{\xi \mu}^{-}(t)=\sum_{\lambda} 2^{\frac{\ell(\xi)+\delta(\xi)}{2}} g_{\xi \lambda} \gamma_{\lambda \mu}(t)
$$

Then by Theorem 2.3 we obtain

$$
\gamma_{\xi \mu}^{-}(t)=\sum_{\lambda} 2^{\frac{\ell(\xi)+\delta(\xi)}{2}} g_{\xi \lambda} K_{\lambda \mu}(t)=\sum_{\lambda} 2^{-\frac{\ell(\xi)-\delta(\xi)}{2}} b_{\xi \lambda} K_{\lambda \mu}(t) .
$$

This together with Proposition 2.5 proves Theorem $\mathrm{B}(2)$.

The interpretation of spin Kostka polynomials as $q$-weight multiplicity can take another form.

Proposition 3.3 Suppose $\xi \in \mathcal{S P}$ and $\mu \in \mathcal{P}$ with $\ell(\xi) \leq n$ and $\ell(\mu) \leq n$. Then we have

$$
K_{\xi \mu}^{-}(t)=2^{\frac{\ell(\xi)-\delta(\xi)}{2}}\left[e^{\mu}\right] \frac{\prod_{\alpha \in \Delta^{+}}\left(1-e^{-\alpha}\right)}{\prod_{\alpha \in \Delta^{+}}\left(1-t e^{-\alpha}\right)} \operatorname{ch} V(\xi) .
$$

Proof It follows from (2.3) that

$$
K_{\lambda \mu}(t)=\left[e^{\mu}\right] \frac{\prod_{\alpha \in \Delta^{+}}\left(1-e^{-\alpha}\right)}{\prod_{\alpha \in \Delta^{+}}\left(1-t e^{-\alpha}\right)} \operatorname{ch} L(\lambda)
$$


for $\lambda \in \mathcal{P}$ with $\ell(\lambda) \leq n$. Hence by Proposition 2.5 and Lemma 3.2 one deduces that

$$
\begin{aligned}
K_{\xi \mu}^{-}(t) & =\sum_{\lambda \vdash n} 2^{\ell(\xi)} g_{\xi \lambda} \cdot\left[e^{\mu}\right] \frac{\prod_{\alpha \in \Delta^{+}}\left(1-e^{-\alpha}\right)}{\prod_{\alpha \in \Delta^{+}}\left(1-t e^{-\alpha}\right)} \operatorname{ch} L(\lambda) \\
& =\left[e^{\mu}\right] \frac{\prod_{\alpha \in \Delta^{+}}\left(1-e^{-\alpha}\right)}{\prod_{\alpha \in \Delta^{+}}\left(1-t e^{-\alpha}\right)} \sum_{\lambda \in \mathcal{P}, \ell(\lambda) \leq n} 2^{\ell(\xi)} g_{\xi \lambda} \operatorname{ch} L(\lambda) \\
& =2^{\frac{\ell(\xi)-\delta(\xi)}{2}}\left[e^{\mu}\right] \frac{\prod_{\alpha \in \Delta^{+}}\left(1-e^{-\alpha}\right)}{\prod_{\alpha \in \Delta^{+}}\left(1-t e^{-\alpha}\right)} \operatorname{ch} V(\xi)
\end{aligned}
$$

\section{Spin Hall-Littlewood and spin Macdonald polynomials}

In this section, we introduce the spin Hall-Littlewood polynomials and establish their main properties. We also formulate the $q, t$-generalizations of spin Kostka polynomials and Macdonald polynomials.

\subsection{A commutative diagram}

Recall a homomorphism $\varphi[13$, III, Sect. 8, Example 10] defined by

$$
\begin{aligned}
& \varphi: \Lambda \longrightarrow \Gamma, \\
& \varphi\left(p_{r}\right)= \begin{cases}2 p_{r}, & \text { for } r \text { odd } \\
0, & \text { otherwise },\end{cases}
\end{aligned}
$$

where $p_{r}$ denotes the $r$ th power sum symmetric function. Denote

$$
H(t)=\sum_{n \geq 0} h_{n} t^{n}=\prod_{i} \frac{1}{1-x_{i} t}=\exp \left(\sum_{r \geq 1} \frac{p_{r} t^{r}}{r}\right) .
$$

Noting that $Q(t)$ from (2.5) can be rewritten as

$$
Q(t)=\exp \left(2 \sum_{r \geq 1, r \text { odd }} \frac{p_{r} t^{r}}{r}\right)
$$

we obtain

$$
\varphi(H(t))=Q(t)
$$

Hence, we have $\varphi\left(h_{n}\right)=q_{n}$ for all $n$, and

$$
\varphi\left(h_{\mu}\right)=q_{\mu}, \quad \forall \mu \in \mathcal{P} .
$$

For each $n \geq 0$, we define a functor

$$
\Phi_{n}: S_{n}-\bmod \longrightarrow \mathcal{H}_{n}-\operatorname{smod}
$$


by sending $M$ to ind ${ }_{\mathbb{C} S_{n}}^{\mathcal{H}_{n}} M$. Such a sequence of functors $\left\{\Phi_{n}\right\}$ induces a $\mathbb{Z}$-linear map on the Grothendieck group level:

$$
\Phi: R \longrightarrow R^{-}
$$

by letting $\Phi([M])=\left[\Phi_{n}(M)\right]$ for $M \in S_{n}$-mod. We shall show that the map $\Phi: R \rightarrow$ $R^{-}$(or the sequence $\left\{\Phi_{n}\right\}$ ) is a categorification of $\varphi: \Lambda \rightarrow \Gamma$.

Recall that $R$ carries a natural Hopf algebra structure with multiplication given by induction and comultiplication given by restriction [23]. In the same fashion, we can define a Hopf algebra structure on $R^{-}$by induction and restriction. On the other hand, $\Lambda_{\mathbb{Q}} \cong \mathbb{Q}\left[p_{1}, p_{2}, p_{3}, \ldots\right]$ is naturally a Hopf algebra, where each $p_{r}$ is a primitive element, and $\Gamma_{\mathbb{Q}} \cong \mathbb{Q}\left[p_{1}, p_{3}, p_{5}, \ldots\right]$ is naturally a Hopf subalgebra of $\Lambda_{\mathbb{Q}}$. The characteristic map ch : $R_{\mathbb{Q}} \rightarrow \Lambda_{\mathbb{Q}}$ is an isomorphism of Hopf algebras (cf. [23]). A similar argument easily leads to the following.

Lemma 4.1 The map $\mathrm{ch}^{-}: R_{\mathbb{Q}}^{-} \rightarrow \Gamma_{\mathbb{Q}}$ is an isomorphism of Hopf algebras.

Proposition 4.2 The map $\Phi: R_{\mathbb{Q}} \rightarrow R_{\mathbb{Q}}^{-}$is a homomorphism of Hopf algebras. Moreover, we have the following commutative diagram of Hopf algebras:

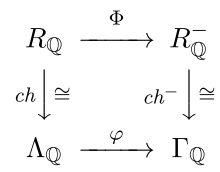

Proof Using (3.2) and (4.3) we have

$$
\varphi\left(\operatorname{ch}\left(\operatorname{ind}_{\mathbb{C} S_{\mu}}^{\mathbb{C} S_{n}} \mathbf{1}\right)\right)=q_{\mu}
$$

On the other hand, it follows by (3.5) that

$$
\operatorname{ch}^{-}\left(\Phi\left(\operatorname{ind}_{\mathbb{C} S_{\mu}}^{\mathbb{C} S_{n}} \mathbf{1}\right)\right)=\operatorname{ch}^{-}\left(\operatorname{ind}_{\mathbb{C} S_{\mu}}^{\mathcal{H} C_{n}} \mathbf{1}\right)=q_{\mu}
$$

This establishes the commutative diagram on the level of linear maps, since $R_{n}$ has a basis given by the characters of the permutation modules ind $\mathbb{C S}_{\mu} \mathbf{C} S_{n}$ for $\mu \in \mathcal{P}_{n}$.

It can be verified easily that $\varphi: \Lambda_{\mathbb{Q}} \rightarrow \Gamma_{\mathbb{Q}}$ is a homomorphism of Hopf algebras. Let us check that $\varphi$ commutes with the comultiplication $\Delta$.

$$
\begin{aligned}
& \Delta\left(\varphi\left(p_{r}\right)\right)=\Delta\left(2 p_{r}\right)=2\left(p_{r} \otimes 1+1 \otimes p_{r}\right)=(\varphi \otimes \varphi)\left(\Delta\left(p_{r}\right)\right), \quad \text { for odd } r . \\
& \Delta\left(\varphi\left(p_{r}\right)\right)=0=(\varphi \otimes \varphi)\left(\Delta\left(p_{r}\right)\right), \quad \text { for even } r .
\end{aligned}
$$

Since both ch and $\mathrm{ch}^{-}$are isomorphisms of Hopf algebras, it follows from the commutativity of (4.4) that $\Phi: R_{\mathbb{Q}} \rightarrow R_{\mathbb{Q}}^{-}$is a homomorphism of Hopf algebras. 


\subsection{Spin Hall-Littlewood functions}

Denote by $H_{\mu}(x ; t)$ the basis of $\Lambda$ dual to the Hall-Littlwood functions $P_{\mu}(x ; t)$ with respect to the standard inner product $(\cdot, \cdot)$ on $\Lambda$ such that Schur functions form an orthonormal basis. It follows by the Cauchy identity and (2.1) that

$$
\begin{aligned}
\prod_{i, j} \frac{1}{1-x_{i} y_{j}} & =\sum_{\mu} H_{\mu}(x ; t) P_{\mu}(y ; t), \\
H_{\mu}(x ; t) & =\sum_{\lambda \in \mathcal{P}} K_{\lambda \mu}(t) s_{\lambda}(x) .
\end{aligned}
$$

Recall that in $\lambda$-ring formalism, the symmetric functions in $x(1-t)$ are defined in terms of $p_{k}((1-t) x)=\left(1-t^{k}\right) p_{k}(x)$. Actually, the symmetric functions $P_{\mu}(x ; t)$ and $H_{\mu}(x ; t)$ are related to each other as (cf. [3])

$$
P_{\mu}(x ; t)=\frac{1}{b_{\mu}(t)} H_{\mu}((1-t) x ; t),
$$

where $b_{\mu}(t)=\prod_{i \geq 1} \prod_{k=1}^{m_{i}(\mu)}\left(1-t^{k}\right)$ and $m_{i}(\mu)$ denotes the number of times $i$ occurs as a part of $\mu$.

Definition 4.3 Define the spin Hall-Littlewood function $H_{\mu}^{-}(x ; t)$ for $\mu \in \mathcal{P}$ by

$$
H_{\mu}^{-}(x ; t)=\sum_{\xi \in \mathcal{S} \mathcal{P}} 2^{-\ell(\xi)} K_{\xi \mu}^{-}(t) Q_{\xi}(x)
$$

For $\lambda \in \mathcal{P}$, let $S_{\lambda} \in \Gamma$ be the determinant (cf. [13, III, Sect. 8, 7(a)])

$$
S_{\lambda}=\operatorname{det}\left(q \lambda_{i}-i+j\right)
$$

It follows by the Jacobi-Trudi identity for $s_{\lambda}$ and (4.3) that

$$
\varphi\left(s_{\lambda}\right)=S_{\lambda}
$$

Applying $\varphi$ to the Cauchy identity $\prod_{i, j} \frac{1}{1-x_{i} y_{j}}=\sum_{\lambda \in \mathcal{P}} s_{\lambda}(x) s_{\lambda}(y)$ and using (4.2) with $t=y_{i}$, we obtain

$$
\prod_{i, j \geq 1} \frac{1+x_{i} y_{j}}{1-x_{i} y_{j}}=\sum_{\lambda \in \mathcal{P}} S_{\lambda}(x) s_{\lambda}(y) .
$$

It follows by the commutative diagram (4.4) and (4.9) that $S_{\lambda}(x)=\operatorname{ch}\left(\mathcal{C}_{n} \otimes S^{\lambda}\right)$. This recovers and provides a representation theoretic context for [13, III, Sect. 8, 7(c)], as the Clifford algebra $\mathcal{C}_{n}$ is isomorphic to the exterior algebra $\wedge\left(\mathbb{C}^{n}\right)$ as $S_{n}$-modules. 
Theorem 4.4 The spin Hall-Littlewood functions $H_{\mu}^{-}(x ; t)$ for $\mu \in \mathcal{P}$ satisfy the following properties:

(1) $\varphi\left(H_{\mu}(x ; t)\right)=H_{\mu}^{-}(x ; t)$.

(2) $H_{\mu}^{-}(x ; 1)=q_{\mu}(x)$.

(3) $H_{\mu}^{-}(x ; 0)=S_{\mu}(x)$.

(4) $H_{\mu}^{-}(x ;-1)= \begin{cases}Q_{\mu}(x), & \text { if } \mu \in \mathcal{S P}, \\ 0, & \text { otherwise. }\end{cases}$

(5) $H_{\mu}^{-}(x ; t) \in \mathbb{Z}[t] \otimes_{\mathbb{Z}} \Gamma, \forall \mu \in \mathcal{P} ;\left\{H_{\xi}^{-}(x ; t) \mid \xi \in \mathcal{S P}\right\}$ forms a basis of $\mathbb{Z}[t] \otimes_{\mathbb{Z}} \Gamma$.

(6) $\prod_{i, j} \frac{1+x_{i} y_{j}}{1-x_{i} y_{j}}=\sum_{\mu \in \mathcal{P}} H_{\mu}^{-}(x ; t) P_{\mu}(y ; t)$.

Proof By (4.10) and the Cauchy identity for Schur $Q$-functions, we have

$$
\sum_{\xi \in \mathcal{S} \mathcal{P}} 2^{-\ell(\xi)} Q_{\xi}(x) Q_{\xi}(y)=\sum_{\lambda \in \mathcal{P}} S_{\lambda}(x) s_{\lambda}(y)
$$

Substituting with $Q_{\xi}(y)=\sum_{\lambda \in \mathcal{P}} 2^{\ell(\xi)} g_{\xi \lambda} s_{\lambda}(y)$ in the above equation, we obtain

$$
S_{\lambda}(x)=\sum_{\xi \in \mathcal{S} \mathcal{P}} g_{\xi \lambda} Q_{\xi}(x) .
$$

Part (1) can now be proved using (4.6), (4.9), (4.11), Proposition 2.5, and (4.8):

$$
\begin{aligned}
\varphi\left(H_{\mu}(x ; t)\right) & =\sum_{\lambda \in \mathcal{P}} K_{\lambda \mu}(t) S_{\lambda}(x) \\
& =\sum_{\xi \in \mathcal{S} \mathcal{P}, \lambda \in \mathcal{P}} g_{\xi \lambda} K_{\lambda \mu}(t) Q_{\xi}(x) \\
& =\sum_{\xi \in \mathcal{S} \mathcal{P}} 2^{-\ell(\xi)} K_{\xi \mu}^{-}(t) Q_{\xi}(x) \\
& =H_{\mu}^{-}(x ; t) .
\end{aligned}
$$

Since $H_{\mu}(x ; 0)=s_{\mu}$ and $H_{\mu}(x ; 1)=h_{\mu}$, (2) and (3) follow from (4.3), (4.9), and (1). Also, (4) follows by Theorem A(4) and the definition of $H_{\mu}^{-}(x ; t)$.

We have $2^{-\ell(\xi)} K_{\xi \mu}^{-}(t) \in \mathbb{Z}[t]$ by Theorem A(3) and $Q_{\xi}(x) \in \Gamma$, and hence by (4.8), $H_{\mu}^{-}(x ; t) \in \mathbb{Z}[t] \otimes_{\mathbb{Z}} \Gamma$. By (4.8) and Theorem $\mathrm{A}(1)(3)$, the transition matrix between $\left\{H_{\xi}^{-}(x ; t) \mid \xi \in \mathcal{S P}\right\}$ and $\left\{Q_{\xi}(x) \mid \xi \in \mathcal{S P}\right\}$ is unital upper triangular with entries in $\mathbb{Z}[t]$. Therefore, $\left\{H_{\xi}^{-}(x ; t) \mid \xi \in \mathcal{S P}\right\}$ forms a basis of $\mathbb{Z}[t] \otimes_{\mathbb{Z}} \Gamma$ since so does $\left\{Q_{\xi}(x) \mid \xi \in \mathcal{S P}\right\}$. This proves (5).

(6) follows by applying the map $\varphi$ to both sides of (4.5) in $x$ variables and using (4.2) and (1). 
4.3 Spin Macdonald polynomials and spin $q$, $t$-Kostka polynomials

Denote by $H_{\lambda}(x ; q, t)$ the normalized Macdonald polynomials, which is related to the Macdonald integral form $J_{\lambda}(x ; q, t)$ by

$$
H_{\lambda}(x ; q, t)=J(x /(1-t) ; q, t)
$$

in $\lambda$-ring notation (cf. [4, 13]). Inspired by Theorem 4.4(1), we make the following.

Definition 4.5 The spin Macdonald polynomials $H_{\mu}^{-}(x ; q, t)$ for $\mu \in \mathcal{P}$ is given by

$$
H_{\mu}^{-}(x ; q, t)=\varphi\left(H_{\mu}(x ; q, t)\right) .
$$

The spin $q, t$-Kostka polynomials $K_{\xi \mu}^{-}(q, t)$ for $\xi \in \mathcal{S P}$ and $\mu \in \mathcal{P}$ are given by

$$
H_{\mu}^{-}(x ; q, t)=\sum_{\xi \in \mathcal{S} \mathcal{P}} 2^{-\ell(\xi)} K_{\xi \mu}^{-}(q, t) Q_{\xi}(x) .
$$

Compare with (4.6) for Kostka polynomials and (4.8) for spin Kostka polynomials. The classical $q, t$-Kostka polynomial $K_{\lambda \mu}(q, t)$ can be characterized as follows:

$$
H_{\mu}(x ; q, t)=\sum_{\lambda \in \mathcal{P}} K_{\lambda \mu}(q, t) s_{\lambda}(x) .
$$

According to Garsia and Haiman $[4,6]$, there is a $\mathbb{Z}_{+} \times \mathbb{Z}_{+}$-graded regular representation $R_{\mu}$ of $S_{n}$, parameterized by $\mu \in \mathcal{P}_{n}$ :

$$
R_{\mu}=\bigoplus_{i, j \geq 0} R_{\mu}^{i, j}
$$

such that

$$
\sum_{i, j \geq 0} \operatorname{dim}_{\operatorname{Hom}}^{S_{n}}\left(S^{\lambda}, R_{\mu}^{i, j}\right) q^{j} t^{i}=K_{\lambda \mu}\left(q, t^{-1}\right) t^{n(\mu)} .
$$

In particular, this established a conjecture of Macdonald [13] that $K_{\lambda \mu}(q, t) \in$ $\mathbb{Z}_{+}[q, t]$.

For $\mu \in \mathcal{P}_{n}$ we consider the doubly graded $\mathcal{H}_{n}$-module $\Phi_{n}\left(R_{\mu}\right)=\mathcal{C}_{n} \otimes R_{\mu}$, and set

$$
C_{\xi \mu}^{-}(q, t):=\sum_{i, j \geq 0} \operatorname{dim} \operatorname{Hom}_{\mathcal{H}_{n}}\left(D^{\xi}, \mathcal{C}_{n} \otimes R_{\mu}^{i, j}\right) q^{j} t^{i}
$$

Proposition 4.6 The following identities hold for $\xi \in \mathcal{S P}$ and $\mu \in \mathcal{P}$ :

$$
\begin{aligned}
& K_{\xi \mu}^{-}(q, t)=\sum_{\lambda \in \mathcal{P}} b_{\xi \lambda} K_{\lambda \mu}(q, t), \\
& C_{\xi \mu}^{-}(q, t)=2^{-\frac{\ell(\xi)-\delta(\xi)}{2}} K_{\xi \mu}^{-}\left(q, t^{-1}\right) t^{n(\mu)} .
\end{aligned}
$$


Proof The identity (4.14) follows by applying the map $\varphi$ to (4.12) and using (4.9) and (4.11).

Suppose $\xi \in \mathcal{S P}_{n}$ and $\mu \in \mathcal{P}_{n}$. We compute by Lemma 3.1 and (4.13) that

$$
\begin{aligned}
C_{\xi \mu}^{-}(q, t) & =\sum_{i, j \geq 0} q^{j} t^{i} \operatorname{dim} \operatorname{Hom}_{\mathcal{H}_{n}}\left(D^{\xi}, \operatorname{ind}_{\mathbb{C} S_{n}}^{\mathcal{H}_{n}} R_{\mu}^{i, j}\right) \\
& =\sum_{\lambda \in \mathcal{P}_{n}} K_{\lambda \mu}\left(q, t^{-1}\right) t^{n(\mu)} \operatorname{dim}_{\operatorname{Hom}_{\mathcal{H}_{n}}}\left(D^{\xi}, \operatorname{ind}_{\mathbb{C} S_{n}}^{\mathcal{H}_{n}} S^{\lambda}\right) \\
& =2^{-\frac{\ell(\xi)-\delta(\xi)}{2}} \sum_{\lambda \in \mathcal{P}_{n}} b_{\xi \lambda} K_{\lambda \mu}\left(q, t^{-1}\right) t^{n(\mu)} .
\end{aligned}
$$

The identity (4.15) follows from this and (4.14).

Note that $K_{\xi \mu}^{-}(0, t)=K_{\xi \mu}^{-}(t)$, and $C_{\xi \mu}^{-}(1,1)=2^{-\frac{\ell(\xi)-\delta(\xi)}{2}} K_{\xi \mu}^{-}(1,1)$ is the degree of $D^{\xi}$. We leave it to the reader to formulate further properties of spin Macdonald polynomials and spin $q, t$-Kostka polynomials.

\subsection{Discussions and open questions}

Let $\left\{\widehat{H}_{\xi}(x ; t) \mid \xi \in \mathcal{S P}\right\}$ be the basis dual to $\left\{H_{\xi}^{-}(x ; t) \mid \xi \in \mathcal{S P}\right\}$ in $\Gamma$ with respect to the inner product $\left\langle Q_{\xi}, Q_{\zeta}\right\rangle=2^{\ell(\xi)} \delta_{\xi \zeta}$ for $\xi, \zeta \in \mathcal{S P}$, or equivalently,

$$
\prod_{i, j} \frac{1+x_{i} y_{j}}{1-x_{i} y_{j}}=\sum_{\xi \in \mathcal{S} \mathcal{P}} H_{\xi}^{-}(x ; t) \widehat{H}_{\xi}(y ; t) .
$$

It follows that

$$
\begin{aligned}
\prod_{i, j} \frac{1+x_{i} y_{j}}{1-x_{i} y_{j}} \frac{1-t x_{i} y_{j}}{1+t x_{i} y_{j}} & =\sum_{\xi \in \mathcal{S} \mathcal{P}} H_{\xi}^{-}(x(1-t) ; t) \widehat{H}_{\xi}(y ; t) \\
& =\sum_{\xi \in \mathcal{S} \mathcal{P}} H_{\xi}^{-}(x ; t) \widehat{H}_{\xi}(y(1-t) ; t) .
\end{aligned}
$$

However, the spin analogue of the relation (4.7), i.e., a similar relation for $H_{\xi}^{-}(x ; t)$ and its dual basis $\widehat{H}_{\xi}(x ; t)$ does not hold, as can be shown by examples for $n=3$.

In the case $\xi=(n)$, we have

$$
H_{(n)}^{-}((1-t) x ; t)=q_{n}((1-t) x)=(-1)^{n} g_{n}(x ; t),
$$

where $g_{n}(x ; t)$ is defined by

$$
\sum_{r \geq 0} g_{r}(x ; t) u^{r}=\prod_{i} \frac{1-u x_{i}}{1+u x_{i}} \cdot \frac{1+t u x_{i}}{1-t u x_{i}} .
$$

Curiously the function $g_{n}(x ; t)$ also appears in our calculation of characters of Hecke-Clifford algebra in [22]. 
According to Lascoux and Schützenberger [11], the Kostka polynomial $K_{\lambda \mu}(t)$ has an interpretation in terms of the charge of semistandard tableaux of shape $\lambda$ and weight $\mu$. This naturally leads to the following.

Question 4.7 Let $\xi \in \mathcal{S} \mathcal{P}, \mu \in \mathcal{P}$ with $|\xi|=|\mu|$. Find a statistics spin charge on marked shifted tableaux, denoted by $\operatorname{sch}(T)$, such that $K_{\xi \mu}^{-}(t)=\sum_{T} t^{\operatorname{sch}(T)}$ where the summation is taken over all marked shifted $\xi$-tableaux $T$ of weight $\mu$. The spin charge is expected to be independent of the marks on the diagonal of a shifted tableau to account for the factor $2^{\ell(\xi)}$ for $K_{\xi \mu}^{-}(t)$.

A possible approach toward spin charge would be using the quantum affine queer algebra introduced by Chen and Guay [2].

Example 4.8 The positive integer polynomials $2^{-\ell(\xi)} K_{\xi \mu}^{-}(t)$ for $n=3,4$ are listed in matrix form as follows.

$$
\begin{aligned}
& (n=3) \\
& {\left[\begin{array}{cccc}
\xi \backslash \mu & (3) & (2,1) & \left(1^{3}\right) \\
(3) & 1 & 1+t & 1+t+t^{2}+t^{3} \\
(2,1) & 0 & 1 & t+t^{2}
\end{array}\right]} \\
& (n=4) \\
& {\left[\begin{array}{cccccc}
\xi \backslash \mu & (4) & (3,1) & (2,2) & (2,1,1) & \left(1^{4}\right) \\
(4) & 1 & 1+t & t+t^{2} & 1+t+t^{2}+t^{3} & 1+t+t^{2}+2 t^{3}+t^{4}+t^{5}+t^{6} \\
(3,1) & 0 & 1 & 1+t & 1+2 t+t^{2} & t+2 t^{2}+2 t^{3}+2 t^{4}+t^{5}
\end{array}\right] .}
\end{aligned}
$$

These examples show that the spin Kostka polynomials given in this paper and those by Tudose and Zabrocki [20] using vertex operators are not the same.

Question 4.9 Does there exist a vertex operator interpretation for our version of spin Hall-Littlewood polynomials?

Recall that the Kostka polynomial $K_{\lambda \mu}(t)$ for $\lambda, \mu \in \mathcal{P}$ is symmetric in the sense that

$$
K_{\lambda \mu}(t)=t^{m_{\lambda \mu}} K_{\lambda \mu}\left(t^{-1}\right)
$$

for some $m_{\lambda \mu} \in \mathbb{Z}$. Example 4.8 seems to indicate such a symmetry property for the spin Kostka polynomials as well, as Bruce Sagan suggested to us.

Question 4.10 Does there exist $m_{\xi \mu}^{-} \in \mathbb{Z}$ so that the spin Koskta polynomial $K_{\xi \mu}^{-}(t)$ for $\xi \in \mathcal{S P}, \mu \in \mathcal{P}$ satisfies

$$
K_{\xi \mu}^{-}(t)=t^{m_{\xi \mu}^{-}} K_{\xi \mu}^{-}\left(t^{-1}\right) ?
$$

Finally, the spin $q, t$-analogue deserves to be further studied. 
Question 4.11 Develop systematically a combinatorial theory for the spin Macdonald polynomials and spin $q, t$-Kostka polynomials.

Acknowledgements We thank Naihuan Jing, Bruce Sagan, and Mark Shimozono for helpful discussions and their interest in this work. The first author is partially supported by Excellent young scholars Research Fund of Beijing Institute of Technology. The research of the second author is partially supported by NSF grant DMS-0800280. This paper is partially written up during our visit to Academia Sinica and NCTS (South) in Taiwan, from which we gratefully acknowledge the support and excellent working environment.

\section{References}

1. Brylinski, R.: Limits of weight spaces, Lusztig's $q$-analogs, and fiberings of adjoint orbits. J. Am. Math. Soc. 2, 517-533 (1989)

2. Chen, H., Guay, N.: Twisted affine Lie superalgebra of type $Q$ and quantization of its enveloping superalgebra. Math. Z. (2012). To appear doi:10.1007/s00209-011-0935-2

3. Désarménien, J., Leclerc, B., Thibon, J.: Hall-Littlewood functions and Kostka-Foulkes polynomials in representation theory. Sém. Lothar. Combin. 32, B32c (1994). Approx. 38 pp

4. Garsia, A., Haiman, M.: A graded representation module for Macdonald's polynomials. Proc. Natl. Acad. Sci. USA 90, 3607-3610 (1993)

5. Garsia, A., Procesi, C.: On certain graded $S_{n}$-modules and the $q$-Kostka polynomials. Adv. Math. 94, 82-138 (1992)

6. Haiman, M.: Hilbert schemes, polygraphs, and the Macdonald positivity conjecture. J. Am. Math. Soc. 14, 941-1006 (2001)

7. Jing, N.: Vertex operators and Hall-Littlewood symmetric functions. Adv. Math. 87, 226-248 (1991)

8. Józefiak, T.: A Class of Projective Representations of Hyperoctahedral Groups and Schur Q-functions. Topics in Algebra, Banach Center Publ., vol. 26, Part 2, pp. 317-326. PWN-Polish Scientific Publishers, Warsaw (1990)

9. Kato, S.: Spherical functions and a $q$-analogue of Kostant's weight multiplicity formula. Invent. Math. 66, 461-468 (1982)

10. Kleshchev, A.: Linear and Projective Representations of Symmetric Groups. Cambridge University Press, Cambridge (2005)

11. Lascoux, A., Schützenberger, M.: Sur une conjecture de H.O. Foulkes. C.R. Acad. Sci. Paris Sér. A-B 286, A323-A324 (1978)

12. Lusztig, G.: Singularities, character formulas, and a $q$-analog of weight multiplicities. In: Analysis and Topology on Singular Spaces, II, III (Luminy, 1981). Astérisque, vol. 101-102, pp. 208-229 (1983)

13. Macdonald, I.G.: Symmetric Functions and Hall Polynomials, 2nd edn. Clarendon Press, Oxford (1995)

14. Sagan, B.: Shifted tableaux, Schur $Q$-functions, and a conjecture of R. Stanley. J. Comb. Theory, Ser. A 45, 62-103 (1981)

15. Schur, I.: Über die Darstellung der symmetrischen und der alternierenden Gruppe durch gebrochene lineare Substitutionen. J. Reine Angew. Math. 139, 155-250 (1911)

16. Sergeev, A.: Tensor algebra of the identity representation as a module over the Lie superalgebras $G L(n, m)$ and $Q(n)$. Math. USSR Sb. 51, 419-427 (1985)

17. Shimozono, M., Zabrocki, M.: Hall-Littlewood vertex operators and generalized Kostka polynomials. Adv. Math. 158, 66-85 (2001)

18. Springer, T.: Trigonometrical sums, Green functions of finite groups and representations of Weyl groups. Invent. Math. 36, 173-207 (1976)

19. Stembridge, J.: Shifted tableaux and the projective representations of symmetric groups. Adv. Math. 74, 87-134 (1989)

20. Tudose, G., Zabrocki, M.: A $q$-analog of Schur's $Q$-functions. In: Algebraic Combinatorics and Quantum Groups, pp. 135-161. World Sci., River Edge (2003). arXiv:math/0203046

21. Wan, J., Wang, W.: Spin invariant theory for the symmetric group. J. Pure Appl. Algebra 215, 1569_ $1581(2011)$

22. Wan, J., Wang, W.: Frobenius character formula and spin generic degrees for Hecke-Clifford algebra. arXiv:1201.2457 (2012)

23. Zelevinsky, A.: Representations of Finite Classical Groups. A Hopf Algebra Approach. Lect. Notes in Math., vol. 869. Springer, Berlin (1981) 